\title{
大孔钙铝石块体材料的制备及表征
}

\author{
郭兴忠，蔡晓波，宋 杰，杨 辉 \\ (浙江大学 材料科学与工程学院, 杭州 310027)
}

摘 要: 以无机盐二水氯化钲 $\left(\mathrm{CaCl}_{2} \cdot 2 \mathrm{H}_{2} \mathrm{O}\right)$ 和六水氯化铝 $\left(\mathrm{AlCl}_{3} \cdot 6 \mathrm{H}_{2} \mathrm{O}\right)$ 为原料, 1,2-环氧丙烷 $(\mathrm{PO})$ 为凝胶促进剂, 粘均 分子量 $M_{\mathrm{w}}=100000$ 的聚氧化乙烯(PEO)为相分离诱导剂, 乙二醇为络合剂, 甲酰胺为干燥控制化学添加剂(DCCA), 采用溶胶-凝胶伴随相分离制备了大孔钙铝石块体。利用扫描电镜(SEM)、压录、X 射线衍射(XRD)等测试技术对所 得的块体进行了表征。结果表明: 加入 PEO 能诱导体系发生相分离; PEO 用量和溶剂中醇水比会对多孔结构产生 明显影响; 在合适凝胶温度下, 当加入适量 PEO 和溶剂时, 可以获得共连续大孔结构的钻铝石块体; $1000^{\circ} \mathrm{C}$ 热处理 后, 干凝胶由无定型转变为钻铝石晶型 $\mathrm{Ca}_{12} \mathrm{Al}_{14} \mathrm{O}_{32} \mathrm{Cl}_{2}$, 块体的共连续大孔结构在热处理后被完整保留, 其大孔孔 径分布在 $1 \sim 2 \mu \mathrm{m}$, 孔隙率为 $73.0 \%$; 热处理前后的钙铝石块体均能保持光滑且致密的骨架。

关 键 词: 大孔块体; 钻铝石; 溶胶一凝胶; 相分离

中图分类号: TQ174 文献标识码: A

\section{Preparation and Characterization of Macroporous Mayenite Monoliths}

\author{
GUO Xing-Zhong, CAI Xiao-Bo, SONG Jie, YANG Hui \\ (Schod of Materials Science and Engineering, Zhejiang University, Hangzhou 310027, China)
}

\begin{abstract}
Macroporous mayenite monoliths were successfully prepared via Sol-Gel process accompanied by phase separation using calcium chloride dihydrate and aluminum chloride hexahydrate as raw materials, propylene oxide (PO) as a gelation agent, and poly (ethylene oxide) with average molecular weight of 100,000 as a phase separation inducer. In addition, glycol was used as a chelating agent and formamide as a drying control chemical additive. Macroporous monoliths were characterized by scanning electron microscope(SEM), mercury porosimetry, and X-ray diffraction (XRD). The result shows that the addition of PEO induces phase separation; the amount of PEO and the ratio of ethanol to water have an important effect on porous structure, and monolithic mayenite with co-continuous marcoporous structure is obtained at appropriate addition of PEO and solvent as well as gelation temperature. The dried gels are amorphous, and transform to $\mathrm{Ca}_{12} \mathrm{Al}_{14} \mathrm{O}_{32} \mathrm{Cl}_{2}$ after heat-treatment at $1000^{\circ} \mathrm{C}$ in air, while the co-continuous macropores are retained. The monolith after heat-treatment has narrow pore size distribution of 1-2 $\mu \mathrm{m}$ and porosity of $73.0 \%$. The monoliths before and after heat-treatment possess smooth and dense skeletons.
\end{abstract}

Key words: macroporous monolith; mayenite; Sol-Gel; phase separation

钙铝石 $\left(12 \mathrm{CaO} \cdot 7 \mathrm{Al}_{2} \mathrm{O}_{3}, \mathrm{C} 12 \mathrm{~A} 7\right)$ 是 $\mathrm{CaO}-\mathrm{Al}_{2} \mathrm{O}_{3}$ 二元 体结构, 每个单胞由 118 个原子组成化学式 体系中一种极具应用前景的材料。C $12 \mathrm{~A} 7$ 具有立方晶 $\quad\left[\mathrm{Ca}_{24} \mathrm{Al}_{28} \mathrm{O}_{64}\right]^{4+} \cdot 2 \mathrm{O}^{2-}$, 前一部分是由 12 个晶体学笼状

收稿日期：2014-06-24; 收到修改稿日期：2014-08-16

基金项目: 国家自然科学基金(51372225); 浙江省自然科学基金(LY13B010001)

National Natural Science Foundation of China (51372225); Natural Science Foundation of Zhejiang Province(LY13B010001)

作者简介: 郭兴忠(1974-), 男, 副教授. E-mail: msewj01@zju.edu.cn

通讯作者: 杨 辉, 教授. E-mail: yanghui@zju.edu.cn 
结构组成的带正电结构, 剩下的两个氧原子则占据 12 个 Ca-Al-O 笼中的两个, 成为 “自由氧离子” [1]。尽 管 $\mathrm{C} 12 \mathrm{~A} 7$ 结构本身稳定, 但很多研究表明结构中的自 由氧离子可被其他离子取代 ${ }^{[2-4]}$ 。当自由氧离子被电子 取代时, C12A7 成为一种室温稳定电子化合物 ${ }^{[5-9]}$, 在 电子器件, 电化学等领域有广泛应用前景 ${ }^{[10-11]}$ 。

目前，已有通过固相法 ${ }^{[12]}$ 制备 $\mathrm{C} 12 \mathrm{~A} 7$ 粉体，提 拉法 ${ }^{[5,13]}$ 制备 $\mathrm{C} 12 \mathrm{~A} 7$ 单晶体, 溶胶-凝胶法 ${ }^{[14]}$ 和脉 冲激光沉积法 ${ }^{[6]}$ 制备 $\mathrm{C} 12 \mathrm{~A} 7$ 薄膜的报道。但制备具 有共连续孔结构的多孔钙铝石材料的报道很少。鉴 于多孔材料受到越来越多的关注和应用 ${ }^{[15-16]}$, 多孔 钙铝石的制备研究将很有价值。

Nakanishi 等 ${ }^{[17]}$ 最早报道了硅氧烷体系的溶胶凝胶伴随相分离现象, 建立了含聚合物体系溶胶凝胶伴随相分离的理论判据，并先后制备 $\mathrm{SiO}_{2}$ 、 $\mathrm{TiO}_{2} 、 \mathrm{ZrO}_{2}$ 等 ${ }^{[17-19]}$ 多孔块体。Gash 等 ${ }^{[20-21]}$ 报道了 通过环氧化合物调控无机盐溶胶一凝胶过程制备氧 化物粉体和薄膜等新材料。在该溶胶一凝胶过程中, 环氧化物通过不可逆开环反应与体系中的游离质 子反应，使整个体系的 $\mathrm{pH}$ 均匀提高，促进水合金属 离子的水解和聚合反应。近年来, 结合聚合物诱导 相分离和环氧化物调控溶胶一凝胶过程, Nakanishi 等 ${ }^{[22-24]}$ 报道了 $\mathrm{Al}_{2} \mathrm{O}_{3} 、 \mathrm{LiFePO}_{4} / \mathrm{C}$ 、铁氧化物等多孔 块体的制备。本课题组已采用该方法制备了 $\mathrm{SiO}_{2}$ 、 $\mathrm{ZrO}_{2}$ 、莫来石、堇青石等 ${ }^{[25-28]}$ 多孔块体。与传统金 属醇盐溶胶-凝胶相比, 环氧化合物调控溶胶一凝 胶具有无机前驱体廉价、水解及聚合过程可控等特 点, 已成为溶胶一凝胶伴随相分离制备多孔块体的 新途径。

本工作以无机盐二水氯化钻和六水氯化铝为原 料, 聚氧化乙烯为相分离诱导剂, 水和乙醇为溶剂,
乙二醇为络合剂, 甲酰胺为干燥控制化学添加剂, 环氧丙烷为凝胶促进剂, 采用溶胶-凝胶伴随相分 离制备大孔钙铝石块体, 重点研究了相分离诱导剂 用量，混合溶剂中醇水相对比例以及凝胶温度对钙 铝石块体形貌的影响规律, 优化了大孔钙铝石块体 的制备工艺参数。

\section{1 实验方法}

\section{1 实验过程}

所用试剂均为分析纯试剂, 未标明的试剂均为 分析纯的国药试剂, 各试样的原料组成如表 1 所示。

将化学计量比的二水氯化钙和六水氯化铝(美 国 Aldrich，99\%)溶于去离子水和乙醇的混合溶剂 中, 然后依次加入乙二醇 (EG), 聚氧乙烯(PEO: $M_{\mathrm{w}}=100000$, 中国 Aladdin, AR)和甲酰胺(FA)。在 室温下摚拌 $1 \mathrm{~h}$ 后, 将所得溶液置于冰水混合物中 冷却至 $0^{\circ} \mathrm{C}$, 然后缓慢滴加环氧丙烷 $(\mathrm{PO}$ : 美国 Aldrich, 99.5\%)摚拌 $2 \mathrm{~min}$ 后, 置于不同温度烘箱 中进行凝胶和陈化。陈化 $24 \mathrm{~h}$ 后, 将凝胶置于 $60^{\circ} \mathrm{C}$ 烘箱中干燥 $72 \mathrm{~h}$, 得到钙铝石干胶块体试样。取一 部分试样在 $1000^{\circ} \mathrm{C}$ 下热处理 $2 \mathrm{~h}$, 得到大孔钙铝石 块体。

\section{2 测试方法}

采用场发射扫描电子显微镜(SIRION-100, FEI 公司, 荷兰)观察热处理前后试样自然断面的 微观形貌。采用 X 射线衍射仪(Empyrean 200895, PANalytical B.V., 荷兰)分析热处理前后试样的物 相组成。采用压录仪(AutoPore IV 9510, Micromeritics 仪器, 美国)测试热处理前后试样的大孔 孔径分布。

表 1 试样的原料组成

Table 1 Starting compositions of samples

\begin{tabular}{lcccccc}
\hline & $\begin{array}{c}\left(\mathrm{CaCl}_{2} / \mathrm{AlCl}_{3}\right) \\
/\left(\mathrm{g}^{-1}\right)\end{array}$ & $\begin{array}{c}\left(\mathrm{EtOH} / \mathrm{H}_{2} \mathrm{O}\right) \\
/\left(\mathrm{mL} \cdot \mathrm{mL}^{-1}\right)\end{array}$ & $\begin{array}{c}\mathrm{EG}^{\mathrm{a}} \\
/ \mathrm{mL}\end{array}$ & $\begin{array}{c}\mathrm{FA}^{\mathrm{b}} \\
/ \mathrm{mL}\end{array}$ & $\begin{array}{c}\mathrm{PO}^{\mathrm{c}} \\
/ \mathrm{mL}^{2}\end{array}$ & $\begin{array}{c}\mathrm{PEO}^{\mathrm{d}} \\
/ \mathrm{g}\end{array}$ \\
\hline $\mathrm{CA01}$ & $1.044 / 2.000$ & $3.00 / 3.00$ & 0.40 & 0.50 & 1.50 & 0 \\
$\mathrm{CA02}$ & $1.044 / 2.000$ & $3.00 / 3.00$ & 0.40 & 0.50 & 1.50 & 0.100 \\
$\mathrm{CA03}$ & $1.044 / 2.000$ & $3.00 / 3.00$ & 0.40 & 0.50 & 1.50 & 0.200 \\
$\mathrm{CA04}$ & $1.044 / 2.000$ & $3.00 / 3.00$ & 0.40 & 0.50 & 1.50 & 0.400 \\
$\mathrm{CA05}$ & $1.044 / 2.000$ & $3.50 / 2.50$ & 0.40 & 0.50 & 1.50 & 0.200 \\
$\mathrm{CA06}$ & $1.044 / 2.000$ & $4.00 / 2.00$ & 0.40 & 0.50 & 1.50 & 0.200 \\
$\mathrm{CA07}$ & $1.044 / 2.000$ & $2.50 / 3.50$ & 0.40 & 0.50 & 1.50 & 0.200 \\
$\mathrm{CA08}$ & $1.044 / 2.000$ & $2.00 / 4.00$ & 0.40 & 0.50 & 1.50 & 0.200 \\
\hline
\end{tabular}

${ }^{\mathrm{a}} \mathrm{EG}$ : ethylene glycol; ${ }^{\mathrm{b}} \mathrm{FA}$ : formamide; ${ }^{\mathrm{C} P O}$ : propylene oxide; ${ }^{\mathrm{d}} \mathrm{PEO}$ : poly (ethylene oxide) 


\section{2 结果与讨论}

\subsection{PEO 用量对大孔块体形貌的影响规律}

在本体系中, 氯化钻和氯化铝为原料, PEO 为 相分离诱导剂, $\mathrm{PO}$ 为凝胶促进剂, EG 为络合剂络合 体系中 $\mathrm{Ca}^{2+}$ 离子, 通过溶胶-凝胶伴随相分离过程 得到均匀稳定的湿凝胶。为了保持体系的化学计量 比, 未对凝胶后的湿凝胶进行溶剂置换处理, 而是 通过加入 FA 作为干燥控制添加剂增加骨架强度, 减少凝胶在干燥过程中的收缩 ${ }^{[29]}$

图 1 是不同 PEO 用量制备的钙铝石干凝胶的 SEM 照片。从图 1 可以看出, 当不加入 PEO 时, 体系 几乎不发生相分离, 干凝胶的结构致密 (CA01, 图 1 (a)); 当加入少量 PEO 时, 可得到孔径约为 $0.5 \mu \mathrm{m}$ 的 独立孔结构(CA02, 图 1(b)); 随着 PEO 用量的进一步 增加，体系在微米尺度形成骨架均匀光滑的共连续结 构(CA03, 图 1(c)), 但当 PEO 用量过多时, 大孔结构消 失, 凝胶基体由球形颗粒堆积而成(CA04，图 1(d))。

根据 Nakanishi 建立的初步相分离理论判据,含 有至少一种聚合物的混合体系的相容性可使用弗洛 里一哈金斯晶格理论进行分析，该混合体系的自由 能变化 $\Delta G$ 为 ${ }^{[17]}$ :

$$
\begin{gathered}
\Delta G \propto \mathrm{R} T\left(\varphi_{1} / P_{1} \ln \varphi_{1}+\varphi_{2} / P_{2} \ln \varphi_{2}+\chi \varphi_{1} \varphi_{2}\right) \\
\chi \propto\left(\delta_{1}-\delta_{2}\right)^{2} /\left(K_{\mathrm{B}} T\right)
\end{gathered}
$$

其中 $\varphi_{\mathrm{i}}$ 和 $P_{\mathrm{i}}(\mathrm{i}=1,2)$ 分别是成分 1 和 2 的体积分 数和聚合度, $\mathrm{R}$ 是气体常数, $T$ 是反应温度, $\chi$ 是成分 1 和 2 的相互作用参数, $\delta_{\mathrm{i}}(\mathrm{i}=1,2)$ 是成分 1 和 2 的溶 解参数, $K_{\mathrm{B}}$ 为 Kauri-Butanol 值。当 $\Delta G$ 由负值变为 正值, 即 $\Delta G>0$ 时，混合体系发生相分离。本体系
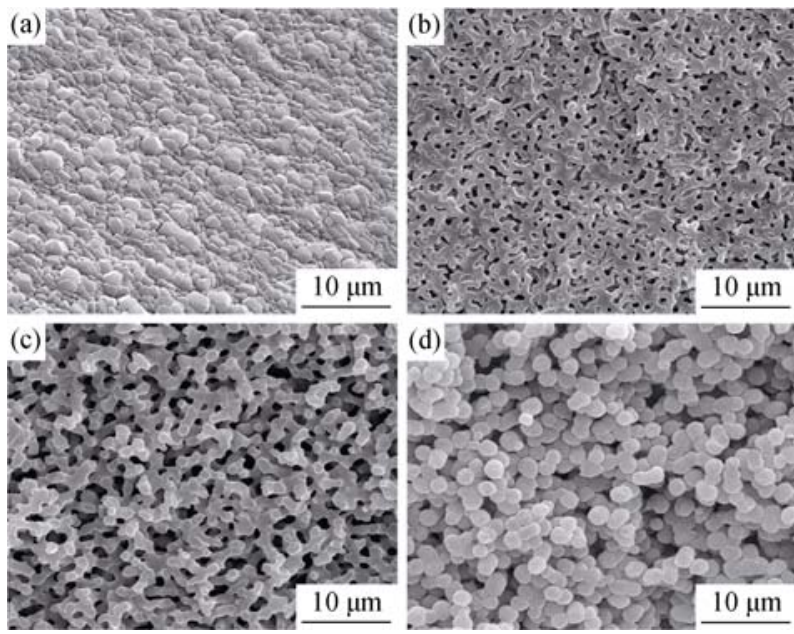

图 1 不同 PEO 用量制备的钻铝石干凝胶的 SEM 照片 Fig. 1 SEM images of gels prepared with varied $W_{\text {PEO }}$ (a) 0 ; (b) $0.100 \mathrm{~g}$; (c) $0.200 \mathrm{~g}$; (d) $0.400 \mathrm{~g}$
中, $\operatorname{PEO}\left(M_{\mathrm{v}}=100000\right)$ 作为相分离诱导剂加入, $\mathrm{PEO}$ 用量的增加增大了体系的相分离趋势。适量的 PEO 可使凝胶保留相分离过程中具有共连续结构的过渡 态，过量的 PEO 则使凝胶保留相分离后期 Ostwald 熟化结构 ${ }^{[15]}$ 。

\section{2 溶剂中醇水比对大孔块体形貌的影响}

采用溶胶-凝胶伴随相分离法制备多孔块体时, 需要精确控制体系的溶剂种类和用量。这是因为溶剂 在相分离后主要形成液相, 包围在凝胶相周围, 这些 液相在后续干燥时蒸发形成孔隙, 溶剂的用量很大程 度上决定了块体孔结构的大小和形貌 ${ }^{[15,17]}$ 。控制体系 溶剂的总体积不变，调整水与乙醇的相对用量 (CA05-CA08)会对大孔形貌产生明显影响。图 2 是醇 水比较高时, 制备的钻铝石干凝胶的 SEM 照片。随着 醇水相对用量的增加，在微米尺度的凝胶形貌由完整 的共连续结构(CA03, 图 2(a)) 向介孔结构(CA05, 图 2(b))转变, 最后形成无孔的致密凝胶(CA06, 图 2(c))。 而体系的凝胶时间只缩短了很少(从 $18 \mathrm{~min}$ 到 $12 \mathrm{~min}$ ), 由此证明这种结构的产生是由相分离过程主导的。

PEO 具有 $\left(-\mathrm{CH}_{2}-\mathrm{CH}_{2}-\mathrm{O}-\right)_{n}$ 结构单元，在水溶液 中有较好的溶解性, 但在乙醇中溶解性较差 ${ }^{[30]}$ 。前期 研究已证明 $\mathrm{Ca}-\mathrm{Al}-\mathrm{O}$ 体系的溶胶一凝胶伴随着相分离 过程, PEO 在相分离后进入溶剂相, 相分离趋势由凝 胶相与 PEO 溶剂相之间的相互作用决定，这与 $\mathrm{Al}_{2} \mathrm{O}_{3}$ 体系相似 ${ }^{[22]}$, 而与传统的 $\mathrm{SiO}_{2}$ 体系不同 ${ }^{[17,25]}$ 。随着 醇水比的提高, PEO 在醇水混合溶剂中的溶解度逐 渐降低, 这减小了 PEO 与凝胶相之间的分离趋势, 即式(1)中 $\chi$ 值减小抑制了相分离，体系凝胶后得到 相分离初期的无孔或介孔形貌。

图 3 是醇水比较低时,制备的钻铝石干凝胶的 SEM 照片，可以发现，随着醇水比的减小，块体微观 形貌由共连续结构(CA03, 图 3(a))经过粗大的骨架 结构(CA07, 图 3(b)) 向大约 $10 \mu \mathrm{m}$ 的独立孔结构 (CA08, 图 3(c))转变。这种结构的变化可由水在溶剂 相和凝胶相之间的分布情况解释: 当水用量进一步 增加时，部分水进入凝胶相，在亚微米尺度发生二次 分相，生成相分离后期形貌，即球形小颗粒，附着在 粗大的骨架上; 当进入凝胶相的水过多时，原有的共 连续结构被破坏，得到被颗粒包围的独立孔结构。

\section{3 凝胶温度对大孔块体形貌的影响规律}

根据相分离理论判据，反应温度也会对体系相 分离产生影响。随着凝胶温度的上升，体系凝胶时间 迅速变短，从 $20^{\circ} \mathrm{C}$ 的 $35 \mathrm{~min}$ 减少到 $80^{\circ} \mathrm{C}$ 的 $2.5 \mathrm{~min}$ 。 图 4 是 CA03 试样在不同凝胶温度得到的干凝胶的 SEM 照片。不同凝胶温度下得到的钙铝石干凝胶块 

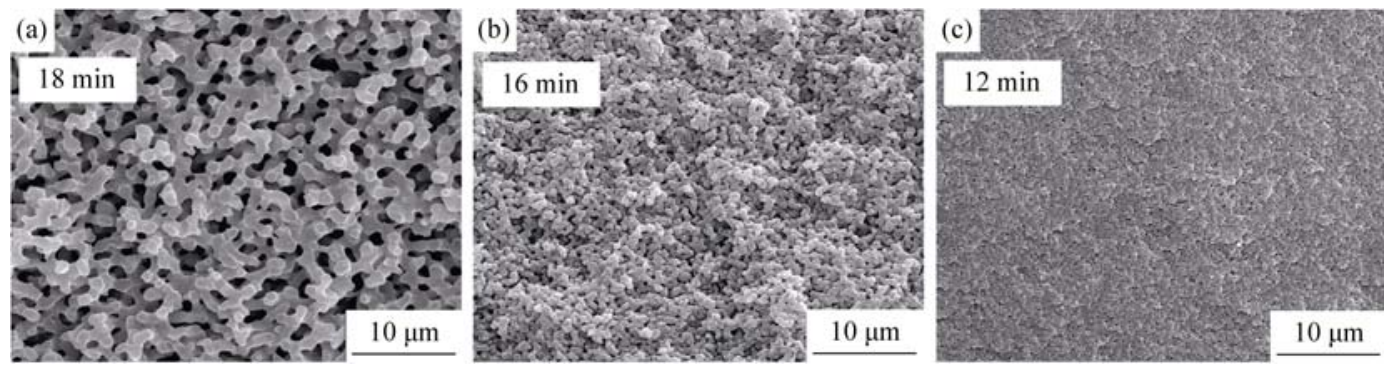

图 2 醇水比较高时制备的钘铝石干凝胶的 SEM 照片

Fig. 2 SEM images of dried gels prepared with high volume ratios of ethanol to water (a) $1 / 1$, (b) $7 / 5$, (c) $2 / 1$. Time inserted each image indicates the length of gelation time
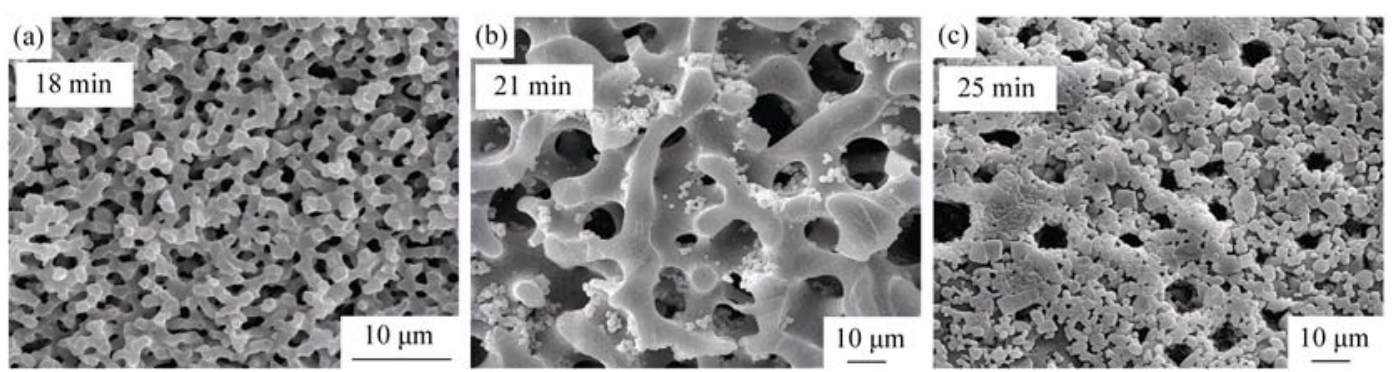

图 3 醇水比较低时制备的钻铝石干凝胶的 SEM 照片

Fig. 3 SEM images of dried gels prepared with low volume ratios of ethanol to water (a) $1 / 1$, (b) $5 / 7$, (c) $1 / 2$. Time inserted each image indicates the length of gelation time

体均具有共连续结构, 但孔结构和骨架变化明显。 当凝胶温度较低时, 块体的大孔孔径较小, 骨架较 细 $\left(20^{\circ} \mathrm{C}\right.$, 图 4(a)); 当凝胶温度适当升高时, 可以得 到共连续孔结构和光滑且致密的骨架 $\left(40{ }^{\circ} \mathrm{C}\right.$, 图 4(b)); 当温度进一步升高时, 骨架变得粗大不均匀, 可以观察到骨架上的颗粒 $\left(60^{\circ} \mathrm{C}\right.$ 和 $80^{\circ} \mathrm{C}$, 图 4(c)、 (d))。当体系温度升高时, 聚合物 PEO 在醇水混合 溶剂中的溶解度上升，体系的相分离趋势加强。同 时, 反应温度升高促进体系的水解和聚合过程, 加 快体系凝胶。在较高的相分离趋势和较快的凝胶速 率共同作用下，由凝胶过程保留的相分离过渡态不
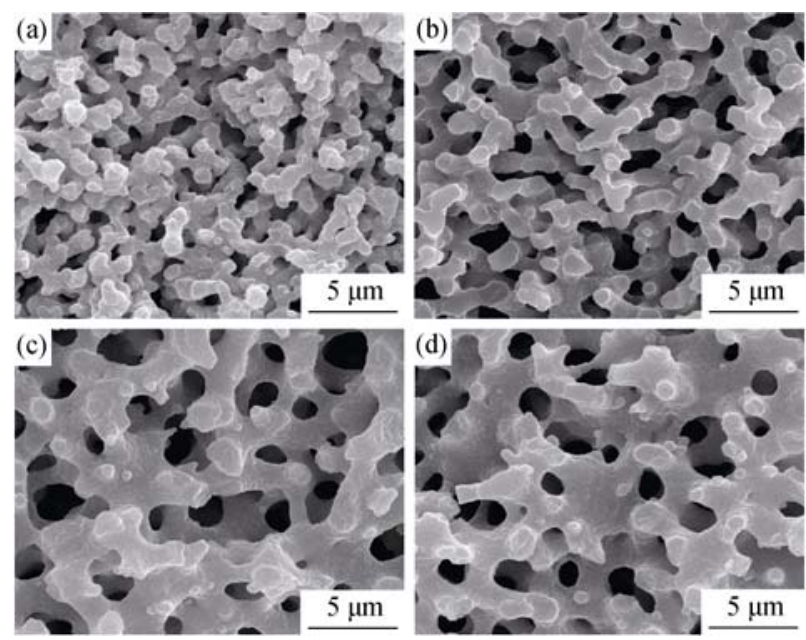

图 4 不同凝胶温度得到的钻铝石干凝胶的 SEM 照片

Fig. 4 SEM images of dried gels prepared at different gelation temperatures

(a) $20^{\circ} \mathrm{C}$; (b) $40^{\circ} \mathrm{C}$; (c) $60^{\circ} \mathrm{C}$; (d) $80^{\circ} \mathrm{C}$
会产生明显的差别。 $20^{\circ} \mathrm{C}$ 时凝胶时间过长, 试样凝 胶后的骨架强度下降, 导致干燥时孔结构收缩, 得 到相对致密的结构。试样在 $60^{\circ} \mathrm{C}$ 和 $80^{\circ} \mathrm{C}$ 凝胶时的形 貌相似, 凝胶时间也较为接近, 这可能是因为反应 温度过高时，体系还未升温至该温度已凝胶。

\section{4 大孔块体的热处理}

对干凝胶块体形貌最佳的试样 CA03 在空气中进 行热处理, 图 5 是试样 CA03 热处理前后的 XRD 图谱。 热处理前的干凝胶是无定型的, $1000^{\circ} \mathrm{C}$ 热处理后形成 了钻铝石晶相 $\mathrm{Ca}_{12} \mathrm{Al}_{14} \mathrm{O}_{32} \mathrm{Cl}_{2}$ ( $\mathrm{PDF} \# 45$-礴 0568)。该物 相不是纯钲铝石相 $\mathrm{Ca}_{12} \mathrm{Al}_{14} \mathrm{O}_{33}$, 这主要是由于使用了 氯盐原料引起的, 氯离子进入钙铝石笼状结构中取代 原有的“自由氧离子”，形成更稳定的晶型 ${ }^{[31]}$ 。

图 6(a)、(b)分别是试样 CA03 热处理前后的宏观

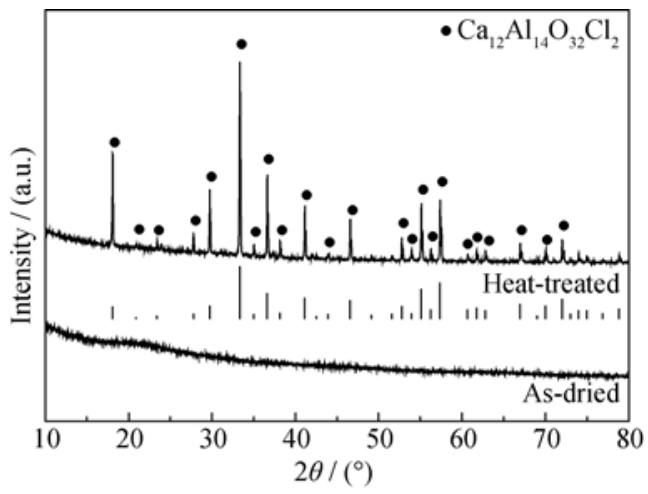

图 5 钙铝石试样 $\mathrm{CA} 03$ 在 $1000^{\circ} \mathrm{C}$ 热处理前后的 XRD 图谱

Fig. 5 XRD patterns of sample CA03 before and after heat-treated at $1000^{\circ} \mathrm{C}$ 

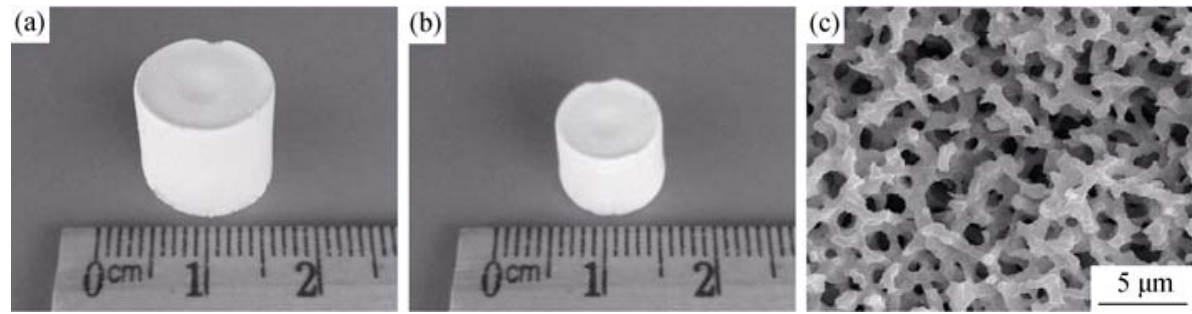

图 6 试样 $\mathrm{CA} 03$ 热处理前(a)和 $1000^{\circ} \mathrm{C}$ 热处理后(b)的宏观形貌和(c)热处理后的 $\mathrm{SEM}$ 照片

Fig. 6 Appearance of sample CA03 (a) before and (b) after heat-treatment at $1000^{\circ} \mathrm{C}$; (c) SEM image of sample CA03 after heat-treatment at $1000^{\circ} \mathrm{C}$

形貌，图 6(c) 是 CA03 热处理后的 SEM 照片。可以 看出, 干燥后的钙铝石块体呈白色, 表面无明显裂 纹; 热处理后的钙铝石块体较热处理前有明显的收 缩, 但仍保持均匀无裂纹的白色块体。热处理后的 钙铝石微观形貌显示, 热处理完整地保留了原有的 共连续结构。共连续骨架产生了收缩, 变得更加光 滑和致密, 大孔结构基本没有变化。

图 7 是压录法测试试样 CA03 热处理前后的大孔 孔径分布, 结果表明, 热处理前后的块体均具有非常 窄的孔径分布, 根据 Nakanishi 的研究 ${ }^{[17]}$, 窄的孔径分 布是体系凝胶过程中发生亚稳态分解相分离的结果。 热处理前试样 CA03 的大孔孔径分布在 1 2 $\mu \mathrm{m}$, 中位 孔径为 $1.8 \mu \mathrm{m}$ 。热处理后块体的中位孔径有少量减小, 孔径分布相对变宽，但孔容明显上升，这与 SEM 分析 结果相符。由表观密度和孔体积计算得到热处理前后 钻铝石块体的孔隙率分别为 59.6\%和 73.0\%。

\section{3 结论}

以无机盐二水氯化钙和六水氯化铝为原料, 环 氧丙烷为凝胶促进剂, 聚氧化乙烯为相分离诱导剂, 乙二醇为络合剂, 甲酰胺为干燥控制化学添加剂,

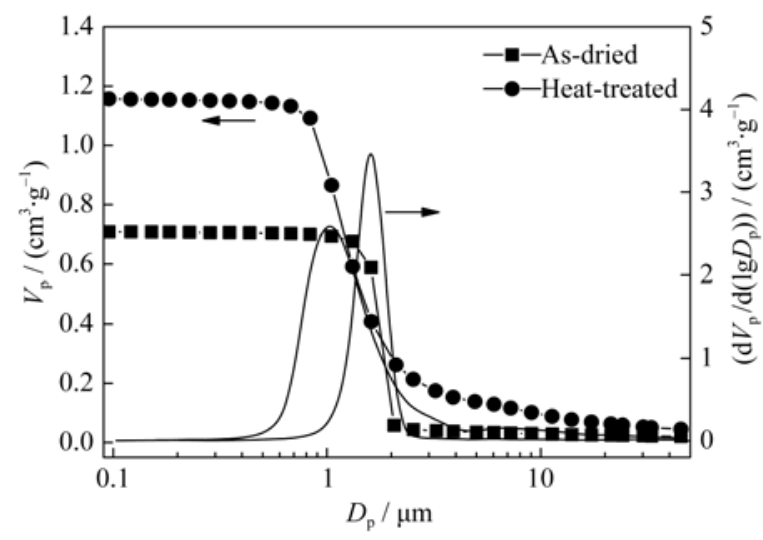

图 7 热处理前后试样 $\mathrm{CA} 03$ 的大孔孔径分布

Fig. 7 Macropore size distribution of sample CA03 before and after heat-treatment at $1000^{\circ} \mathrm{C}$
通过溶胶一凝胶伴随相分离制备了大孔钙铝石块体。

1) PEO 用量和溶剂中醇水比对大孔结构产生明 显影响。在 $40^{\circ} \mathrm{C}$ 凝胶温度下, 使用 $0.200 \mathrm{~g}$ 的 PEO, $3.00 \mathrm{~mL}$ 乙醇和 $3.00 \mathrm{~mL}$ 水的混合溶剂，可以获得具 有共连续大孔结构的钙铝石块体。

2) $1000^{\circ} \mathrm{C}$ 热处理后块体物相由无定型转变为钲 铝石晶相，热处理并未破坏块体的大孔结构，但大 孔孔径分布变宽，分布在 1 2 $\mu \mathrm{m}$, 孔隙率上升至 $73.0 \%$ 。热处理前后大孔块体均具有光滑且致密的共 连续骨架。通过后续的还原处理，该大孔钙铝石块体 材料有望在电化学领域得到广泛应用。

\section{参考文献:}

[1] HOSONO H, ABE Y. Occurrence of superoxide radical ion in crystalline calcium aluminate $12 \mathrm{CaO} \cdot 7 \mathrm{Al}_{2} \mathrm{O}_{3}$ prepared via solidstate reactions. Inorg. Chem., 1987, 26(8): 1192-1195.

[2] JEEVARATNAM J, GLASSER F P, GLASSER L S. Anion substitution and structure of $12 \mathrm{CaO} \cdot 7 \mathrm{Al}_{2} \mathrm{O}_{3}$. J. Am. Ceram. Soc., 1964, 47(2): 105-106.

[3] LI J, HUANG F, WANG L, et al. High density hydroxyl anions in a microporous crystal: $\left[\mathrm{Ca}_{24} \mathrm{Al}_{28} \mathrm{O}_{64}\right]^{4+} \cdot 4\left(\mathrm{OH}^{-}\right)$. Chem. Mater., 2005 , 17: $2771-2774$.

[4] HAYASHI K, HIRANO M, MATSUISHI S, et al. J. Microporous crystal $12 \mathrm{CaO} \cdot 7 \mathrm{Al}_{2} \mathrm{O}_{3}$ encaging abundant $\mathrm{O}^{-}$radicals. J. Am. Chem. Soc., 2002, 124(5): 738-739.

[5] HAYASHI K, MATSUISHI S, KAMIYA T, et al. Light-induced conversion of an insulating refractory oxide into a persistent electronic conductor. Nature, 2002, 419: 462-465.

[6] MIYAKAWA M, HAYASHI K, HIRANO M, et al. Fabtication of highly conductive $12 \mathrm{CaO} \cdot 7 \mathrm{Al}_{2} \mathrm{O}_{3}$ thin films encaging hydride ions by proton implantation. Adv. Mater, 2003, 15(13): 1100-1103.

[7] MATSUISHI S, TODA Y, MIYAKAWA M, et al. High-density electron anions in a nanoporous single crystal. Science, 2003, 301: 626-629.

[8] TODA Y, MATSUISHI S, HAYASHI K, et al. Field emission of electron anions clathrated in subnanometer-sized cages in 
$\left[\mathrm{Ca}_{24} \mathrm{Al}_{28} \mathrm{O}_{64}\right]^{4+} \cdot 4\left(\mathrm{e}^{-}\right)$. Adv. Mater, 2004, 16(8): 685-689.

[9] LI Z, YANG J, HOU J G, et al. Is mayenite without clathrated oxygen an inorganic electride. Angew. Chem., Int. Ed., 2004, 43: 6479-6482.

[10] SUSHKO P V, SHLUGER A L, HIRANO M, et al. From insulator to electride: a theoretical model of nanoporous oxide $12 \mathrm{CaO} \cdot 7 \mathrm{Al}_{2} \mathrm{O}_{3}$. J. Am. Chem. Soc., 2007, 129(4): 942-951.

[11] TODA Y, YANAGI H, IKENAGA E, et al. Work function of a room-temperature, stable electride $\left[\mathrm{Ca}_{24} \mathrm{Al}_{28} \mathrm{O}_{64}\right]^{4+} \cdot 4\left(\mathrm{e}^{-}\right) . A d v \cdot M a$ ter., 2007, 19: 3564-3569.

[12] MATSUISHI S, NOMURA T, HIRANO M, et al. Direct synthesis of powdery inorganic electride $\left[\mathrm{Ca}_{24} \mathrm{Al}_{28} \mathrm{O}_{64}\right]^{4+} \cdot 4\left(\mathrm{e}^{-}\right)$and determination of oxygen stoichiometry. Chem. Mater., 2009, 21: 2589-2591.

[13] KURASHIGE K, TODA Y, MATSTUISHI S, et al. Czochralski growth of $12 \mathrm{CaO} \cdot 7 \mathrm{Al}_{2} \mathrm{O}_{3}$ crystals. Cryst. Growth Design, 2006, 6(7): $1602-1605$.

[14] ZAHEDI M, RAY A K, BARRATT D S. Preparation and crystallization of Sol-Gel C12A7 thin films. J. Phys. D: Appl. Phys., 2008, 41: 035404-035408.

[15] NAKANISHI K, TANAKA N. Sol-Gel with phase separation hierarchically porous materials optimized for high-performance liquid chromatography separations. Acc. Chem. Res., 2007, 40(9): 863-873.

[16] KITADA A, HASEGAWA G, KOBAYASHI Y, et al. Selective preparation of macroporous monoliths of conductive titanium oxides $\mathrm{Ti}_{n} \mathrm{O}_{2 n-1}(n=2,3,4,6)$. J. Am. Chem. Soc., 2012, 134: 10894-10898.

[17] NAKANISHI K. Pore structure control of silica gels based on phase separation. J. Porous Mater., 1997, 4(2): 67-112.

[18] HASEGAWA G, KANAMORI K, NAKANISHI K, et al. Facile preparation of hierarchically porous $\mathrm{TiO}_{2}$ monoliths. J. Am. Ceram. Soc., 2010, 93(10): 3110-3115.

[19] KONISHI J, FUJITA K, OIWA S, et al. Crystalline $\mathrm{ZrO}_{2}$ monoliths with well-defined macropores and mesostructured skeletons prepared by combining the alkoxy-derived Sol-Gel process accompanied by phase separation and the solvothermal process. Chem. Mater., 2008, 20(6): 2165-2173

[20] GASH A E, TILLOTSON T M, SATCHER J H, et al. Use of ep- oxides in the Sol-Gel synthesis of porous iron(III) oxide monoliths from Fe(III) salts. Chem. Mater, 2001, 13: 999-1007.

[21] GASH A E, SATCHER J H, SIMPSON R L, et al. Strong akaganeite aerogel monoliths using epoxides: synthesis and characterization. Chem. Mater., 2003, 15: 3268-3275.

[22] TOKUDOME Y, FUJITA K, NAKANISHI K, et al. Synthesis of monolithic $\mathrm{Al}_{2} \mathrm{O}_{3}$ with well-defined macropores and mesostructured skeletons via the Sol-Gel process accompanied by phase separation. Chem. Mater., 2007, 19(14): 3393-3398.

[23] HASEGAWA G, ISHIHARA Y, KANAMORI K, et al. Facile preparation of monolithic $\mathrm{LiFePO}_{4} /$ Carbon Composites with well-defined macropores for a lithium-ion battery. Chem. Mater, 2011, 23: 5208-5216.

[24] KIDO Y, NAKANISHI K, MIYASAKA A, et al. Synthesis of monolithic hierarchically porous iron-based xerogels from iron(III) salts via an epoxid-mediated Sol-Gel process. Chem. Mater, 2012, 24: 2071-2077.

[25] GUO X Z, LI W Y, ZHU Y, et al. Macroporous $\mathrm{SiO}_{2}$ monoliths prepared via Sol-Gel process accompanied by phase separation. Acta Phys.-Chim. Sin., 2013, 29(3): 646-652.

[26] GUO X Z, LÜLIN Y X, YANG H. Preparation of macroporous zirconia monoliths via Sol-Gel process accompanied by phase separation. J. Chin. Ceram. Soc., 2014, 42(1): 6-10.

[27] GUO X Z, LI W Y, NAKANISHI K, et al. Preparation of mullite monoliths with well-defined macropores and mesostructured skeletons via the Sol-Gel process accompanied by phase separation. J. Eur. Ceram. Soc., 2013, 33(10): 1967-1974.

[28] GUO X Z, NAKANISHI K, KANAMORI K, et al. Preparation of macroporous cordierite monoliths via the Sol-Gel process accompanied by phase separation. J. Eur. Ceram. Soc., 2014, 34: 817-823.

[29] XU Z J, GAN L H, PANG Y C, et al. Preparation of $\mathrm{Al}_{2} \mathrm{O}_{3}$ bulk aerogels by non-supercritical fluid drying. Acta Phys. -Chim. Sin., 2005, 21(2): 221-224.

[30] SAEKI S, KUWAHARA N, NAKATA M, et al. Upper and lower critical solution temperatures in poly (ethylene glycol) solutions. Polymer, 1976, 17(8): 685-689.

[31] SUN J Q, GONG L, SHEN J, et al. Sol-Gel preparation of porous C12A7-Cl' crystals. Acta Phys.-Chim. Sin., 2010, 26(3): 795-798. 